\title{
CADASIL with Multiorgan Involvement: a Complete Autopsy Examination Report
}

\author{
Nikhil Sangle, J. Richard Baringer, Jennifer Majersik, L. Dana DeWitt
}

Key words: CADASIL, complete autopsy, genetic, Notch3

doi:10.1017/cjn.2015.240

Can J Neurol Sci. 2016; 43: 202-205

Cerebral autosomal dominant arteriopathy with subcortical infarcts and leukoencephalopathy (CADASIL) is a hereditary disease of small blood vessels caused by mutations in the Notch3 gene. Tournier-Lasserve and Bousser described an autosomal dominant syndrome characterized mainly by recurrent strokes and neuroimaging evidence of leukoencephalopathy, studying 45 members of a single family clinically and with magnetic resonance imaging (MRI; $)^{1}$ they were then the first to report the pathologic findings in one of the affected subjects, noting myelin loss and pallor sparing the U-fibers.

Histopathological features of degeneration of the medial smooth muscle cells (SMCs) accompanied by deposition of periodic acid Schiff (PAS)-positive, electron dense granular osmiophilic material (GOM) in the small arteries and arterioles have been predominantly reported in autopsied brains and skin biopsies. ${ }^{2}$ Skin involvement in CADASIL is well known and a documented means of making the diagnosis other than genetic testing. Despite this, extra-neurologic organ involvement in CADASIL has rarely been documented.

\section{Clinical Details}

At the age of 59, the patient, a Caucasian male, was hospitalized because of slurred speech and gait imbalance. His wife thought that she had noticed excess emotionality a couple of years earlier with a changing personality, displaying irritability and compulsiveness. He had an MRI at that time showing "white matter disease'. He developed slurred speech and severe hyper-emotionality with easy crying. A year later he developed left arm and leg weakness and failed a swallow test; a feeding tube was placed. Later he became bedridden requiring hospice care prior to his death. There was no clinical evidence for organ failure or systemic disease. He was a non-smoker, non-drinker, with no history of hypertension, hyperlipidemia, or diabetes. He had a history of migraine headaches in his $30 \mathrm{~s}$ and $40 \mathrm{~s}$. These were complicated by visual disturbances and also occasionally by episodes of numbness of his right face. Family history was significant for asymptomatic parents living to 85 (father) and 95 (mother) years. He had three brothers, all still alive, including a dizygotic twin brother with Parkinson's disease and normal brain imaging.

Magnetic resonance imaging scans in our patient revealed dramatic loss of all the white matter in the cerebral hemispheres with punctate holes (hypointensities) in the centrum semiovale. (Figure 1a \& 1b) The white matter abnormalities extended down into the pons where they were apparent as discrete, scattered, small areas of signal accentuation. Prominent holes (hypointensities were seen on T1-weighted imaging in the corpus callosum on the sagittal views (Figures 1c \& 1d). Magnetic resonance angiography showed no significant arterial abnormalities. Transcranial doppler ultrasound vasoreactivity study with Diamox showed low velocities throughout at baseline and no response to Diamox. This was suggestive of poor autoregulation.

Based on the MRI findings, the patient underwent a skin biopsy and genetic testing, the results of which confirmed the diagnosis of CADASIL. Skin biopsy revealed the presence of multiple PAS-positive, granular, electron dense material within the small arteries/arterioles, with degeneration of the smooth muscle cells. Genetic testing consisted of bi-directional sequencing and polymerase chain reaction testing of the Notch3 gene exons 2 through 24, containing all 34 epidermal growth factor-like repeats of the Notch3 receptor protein. In exon 4, this showed p. Arg153Cys, a DNA sequence alteration in the Notch3 gene known to be a CADASIL disease-associated mutation. In addition, in exon 14, there was polymorphism p.Arg734Arg, a variant of unknown but likely non-pathologic significance as it does not cause loss or gain of cysteine residues. Two of his five adult children each had the same genetic results, with clinical findings also consistent with CADASIL.

\section{Autopsy Findings}

The patient died due to acute bilateral bronchopneumonia, a complication secondary to his recumbence and reduced mobility. At autopsy, the unfixed brain weight was 1550 grams. The whole brain was placed in formalin and cut after two weeks of fixation. Sequential sections through the supratentorium revealed multiple, small, well demarcated cystic lesions, ranging in size from 0.5-1.0 centimeters, within the subcortical white matter, basal ganglia and thalamus. The rest of the brain parenchyma revealed no gross abnormality. The blood vessels at the base of the brain showed a normal configuration of the circle of Willis, with mild atherosclerosis. There was mild biventricular dilation of the heart and congestion of the pulmonary parenchyma.

From the Department of Neurology (JRB, JM, LDD), University of Utah Health Sciences Center, Salt Lake City, Utah, USA, Department of Pathology (NS), London Health Sciences Centre, London, ON, Canada.

Received July 1, 2014. Final Revisions Submitted April 13, 2015.

Correspondence to: Nikhil Sangle, Department of Pathology, Schulich School of

Medicine \& Dentistry, London Health Sciences Centre, 339 Windermere Road, London, ON, Canada N6A 5A5. Email: nikhil.sangle@lhsc.on.ca 

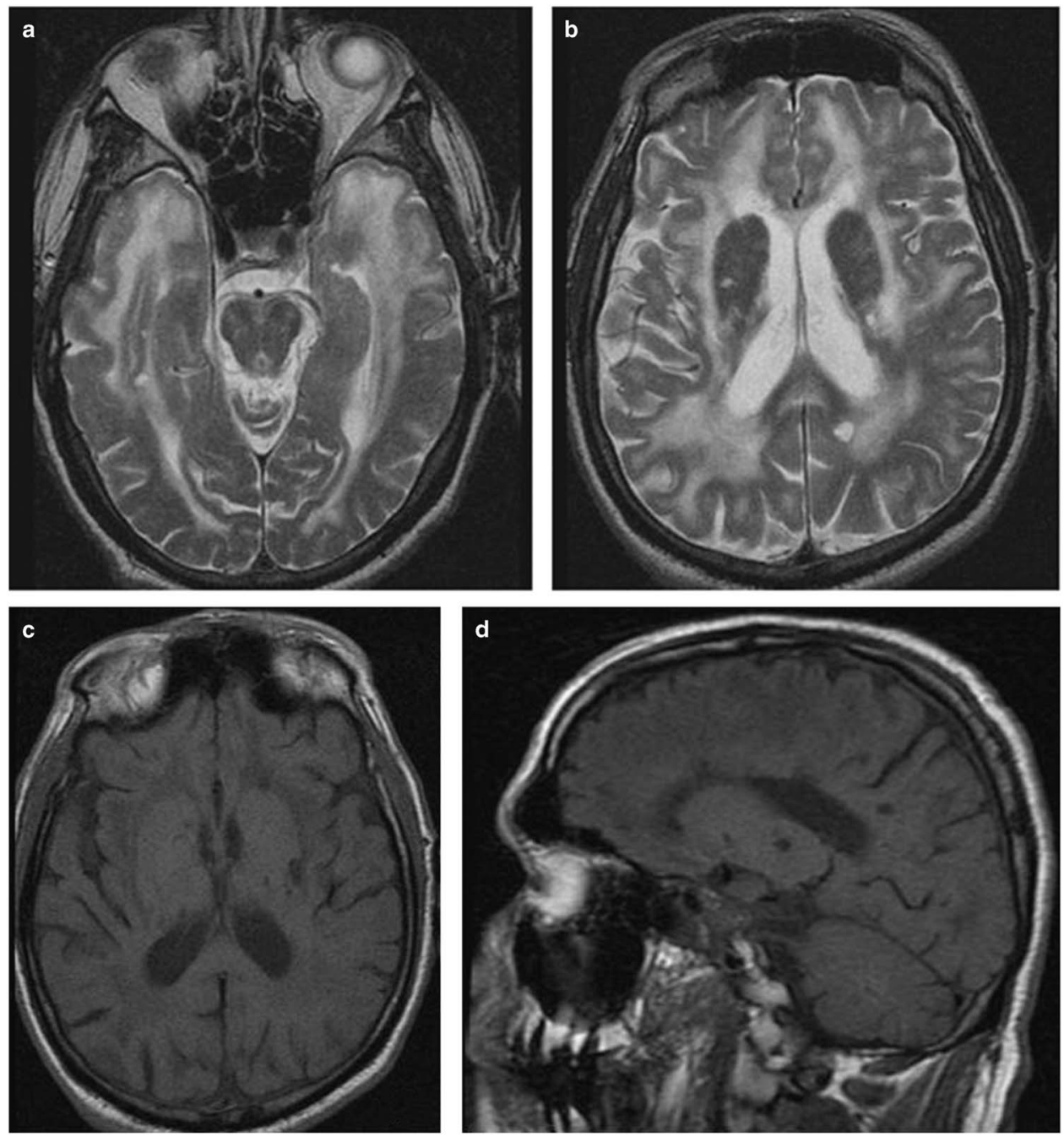

Figure 1: $(a, b)$ Brain magnetic resonance imaging (MRI) T2 showing extensive leukoencephalopathy with marked involvement of anterior temporal lobes; $(c, d)$ Brain MRI T1 showing hypointensities, some in the corpus callosum seen on the sagittal views.

Microscopic examination of the brain revealed multiple old cystic infarcts within the basal ganglia, thalamus and white matter. On routine hematoxylin \& eosin stained sections, no specific diagnostic histopathologic features were identifiable (Figure 2a). On PAS staining, most of the visceral organs, in particular the brain, lungs, spleen, heart, liver, and testes revealed PAS-positive, granular, eosinophilic material within the small and medium sized vessels (Figure 2b). Similar granular material within the walls of paraspinal blood vessels was noted, though the spinal cord tissue was unremarkable. There was degeneration and attenuation of the medial smooth muscle cells and no intimal thickening. The internal elastic lamina and endothelial cells were well preserved. There was no luminal stenosis or occlusion. During autopsy, certain tissues, namely coronary arteries, skin, kidneys, aorta and its branches, and skeletal muscle were preserved in glutaraldehyde fixative for electron microscopic (EM) study. Electron Microscopic examination confirmed the electron-dense, osmiophilic, granular deposits within the vessel walls (Figure 2c). A commercially available antibody for Notch3 gene mutation (clone ab60087, 1:50; abcam) was employed to further characterize the extent of disease. The Notch3 antibody highlighted the osmiophilic, PAS-positive granular material within the blood vessel walls in the majority of visceral organs (Figure 2d). Autopsy sections from uninvolved visceral organs and brain from other cases were used as normal controls.

\section{Discussion}

The patient in our case had the classical mutation of Notch3 gene. This has been a consistent finding in all the reported cases of CADASIL in the literature. The Notch 3 gene has been mapped on chromosome 19p13.1. ${ }^{3}$ Disease-causing mutations of the Notch 3 gene are characterized by a loss or gain of cysteine residues located within the 23 exons (exons 2-24) that encode for the 34 epidermal growth factor (EGF)-like repeat domains of the Notch3 receptor. These missense mutations cause CADASIL in the vast majority of patients. The disease was first reported in European families; however, since 1993 it has been in observed in American, African and Asiatic pedigrees, raising the possibility of it being largely underdiagnosed. 

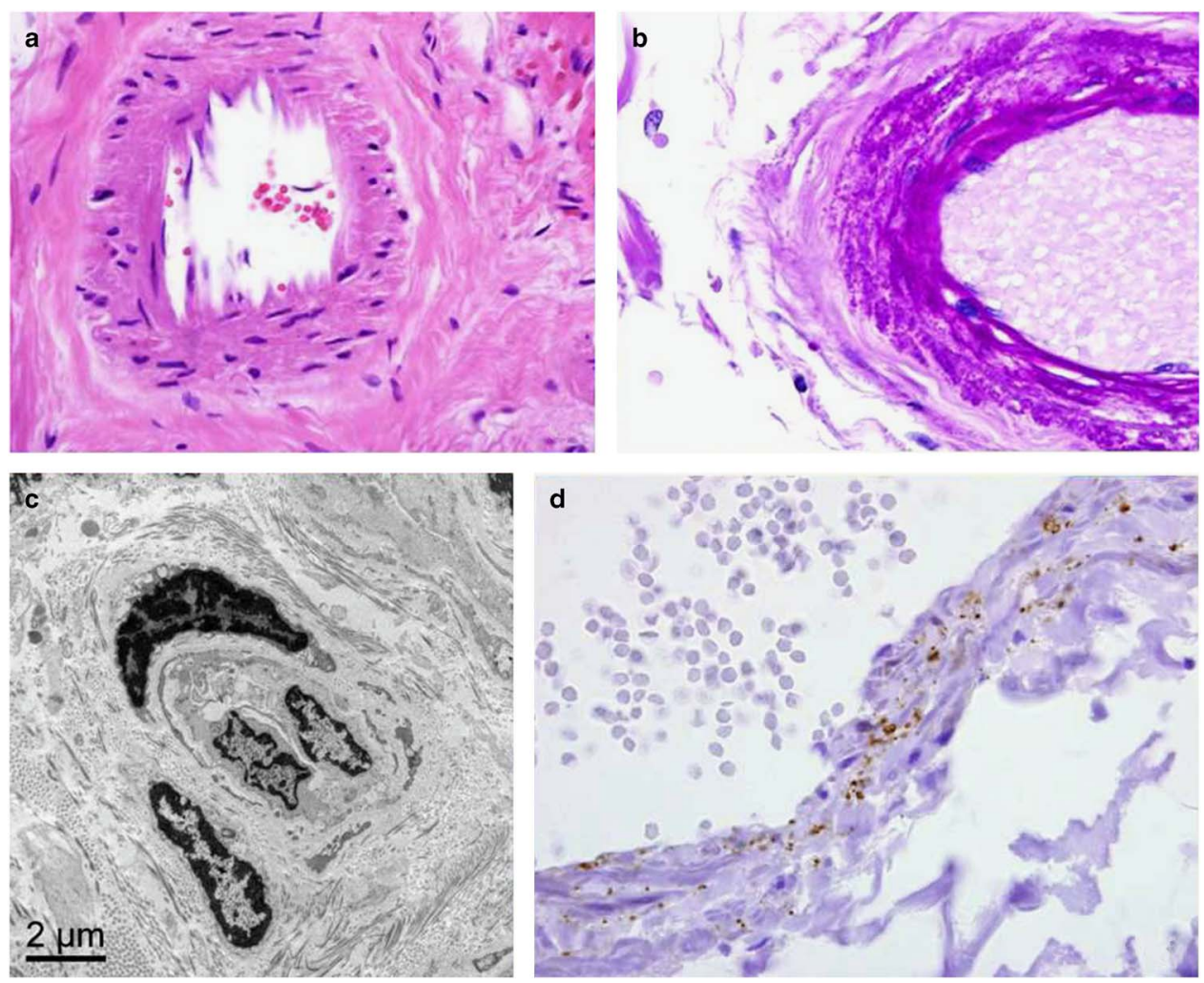

Figure 2: (a) Hematoxylin and eosin stained vessel showing no obvious vascular abnormalities; (b) Periodic acid-Schiff (PAS) staining showing PAS-positive, granular, osmiophilic material within the small and medium sized vessels; (c) Electron microscopic examination showing electron-dense, osmiophilic, granular deposits within the vessel walls; (d) Notch3 antibody highlighting the osmiophilic, PAS-positive granular material within the blood vessel wall.

Patients with CADASIL present clinically with four principle symptoms - migraine with aura, transient ischemic attacks or strokes, psychiatric symptoms, and cognitive decline with vascular dementia. Migraine in CADASIL is characterized by atypical aura with severe visual or sensory disturbances. Hemiplegic aura may occur and might be related to the close localization of gene for hemiplegic migraine. Onset is variable, between 10 and 30 years of age. Ischemic events begin between 30 and 50 years of age, though MRI is able to detect signs of disease years prior to clinical manifestation of disease. Magnetic resonance images show lesions as T2/FLAIR hyperintensity and T1 hypointensity. Lesions are concentrated in subcortical locations, basal ganglia, white matter, and pons. They are noted characteristically to begin in the white matter of the anterior temporal lobes and are often characterized by lacunar syndromes. Other symptoms of CADASIL can include hemorrhagic stroke, primarily microbleeds, which are usually asymptomatic. Psychiatric symptoms are not uncommon with $20 \%$ of CADASIL patients having mood disturbances, with psychotic disorders common. Epileptic seizures, focal or generalized, occur in 6-10\% of patients. Cognitive dysfunction manifests between ages 40 and 70 with $80 \%$ demented by 65 years of age. The clinical manifestations in our case were within the spectrum of clinical symptoms described in various studies. ${ }^{1}$ Our patient had a DNA sequence alteration in the Notch3 gene that is a known CADASIL-associated mutation. Pathological examination confirmed the presence of cystic microinfarcts in the basal ganglia, subcortical region and thalamus.
Skin involvement in CADASIL is well known and a documented means of making the diagnosis other than genetic testing. In a study examining skin biopsies in 131 Finnish, Swedish, and French patients with genetically confirmed CADASIL, the congruence between NOTCH 3 mutations and the presence of GOM in skin biopsies was $100 \%{ }^{4}$ their patients were noted to have 34 different pathogenic mutations. The authors make the point that the diagnostic skin biopsy should include the border zone between deep dermis and upper subcutis where small arterial vessels of correct size are located. Although the visceral organs appeared normal grossly, there was PAS-positive, granular, eosinophilic, electron dense material within the vessel walls of most of the organs, in particular the spleen, testes, heart, liver, and lungs as well as paraspinal vessels. There was no evidence of ischemic infarction or organ-related damage. Electron microscopic evaluation helped confirm the nature of the eosinophilic granular material. Notch 3 antibody is commercially available and was employed in our case to characterize the extent of organ involvement; PAS staining was, however, equally sensitive and effective to highlight the granular material deposited within the vessel walls.

There are other monogenic conditions involving cerebral small vessels and predisposing to ischemic or hemorrhagic stroke and vascular dementia, some of which have other organ involvement. Cerebral autosomal recessive arteriopathy with subcortical infarcts and leukoencephalopathy (CARASIL), COL4A1-related small vessel disease, autosomal dominant retinal vasculopathy 
with cerebral leukoencephalopathy (AD-RVLC), and Fabry's disease are reviewed along with CADASIL in the recently published paper by Federico et al. ${ }^{5}$ Patients with CARASIL have distinctly different pathological features, characterized by intense arteriosclerosis without the deposition of GOM. Hereditary systemic angiopathy (HSA) is associated with cerebral calcifications, retinopathy, progressive nephropathy, hepatopathy. Hereditary endotheliopathy, retinopathy, nephropathy syndrome (HERNS) causes multiple infarcts with leukoencephalopathy associated with retinal and kidney involvement.

The development of cystic brain infarcts and leukoencephalopathy in CADASIL is believed to be secondary to loss of medial smooth muscle cells, which deprives the cerebral arteries of their ability to regulate blood flow, causing impaired autoregulation and resultant ischemic demyelination. If extrapolated to the visceral organs, this deposition of granular material within vessel walls and subsequent medial smooth muscle degeneration could potentially lead to ischemic damage to various organs. The reports documenting this in the literature are very scant, though recently some reports of renal failure have been published. Questions remain as to whether this was due to CADASIL related arteriopathy and why the brain is preferentially involved clinically. It is noted that, although the symptoms of CADASIL are almost exclusively restricted to the central nervous system, GOM deposits are detected all along the vasculature throughout the body

In a review of 38 CADASIL families reported in Japan from 1997-208 (45 patients) only 4 out of 16 of the patients with the documented retinal exams had narrowed retinal arteries. Pathological findings consistent with a diagnosis of CADASIL in a sural nerve biopsy were documented for the first time in 1995. The generalized nature of the vasculopathy was confirmed by documenting GOM covering the vascular smooth muscle cells in cerebral vessels as well as in extracerebral organs (muscle, nerve, skin, small and large intestine, liver, kidney, and heart) in different organs of the index case and in biopsy material (sural nerve, skeletal muscle, and skin) of seven other family members. In the index case they noted fresh necrosis of muscle fibers with vessel walls of epi- and endomysial arteries, arterioles and capillaries. No patches of GOM were seen in the glomerular vessels of the kidney, but they were detected in afferent and efferent arterioles as well as large intestine, liver, kidney and heart. The vessels of the remaining organs were normal.

There have been rare reports of renal failure in patients with CADASIL. Patients with CADASIL having proteinuria, hematuria, and progressive neurological abnormalities have been described. Our patient had suffered from frequent ischemic events and there was evidence of mild renal insufficiency. Magnetic resonance imaging of the brain revealed multiple infarcts and leukoencephalopathy. Histopathological analysis of the kidney revealed degeneration of arterial medial smooth muscle cells and arterial intimal thickening. Electron microscopy revealed electron-dense deposition also in the same region with GOM observed in the extraglomerular mesangial area and around the vascular smooth muscle cells. Genetic analysis of Notch 3 revealed an R141C missense mutation and the patient was diagnosed with CADASIL. In immunohistological analysis, Notch3 stains were positive in vascular smooth muscle cells of the interlobular arteries and both afferent and efferent arterioles and were weakly positive in the glomerular mesangial area. This patient was also found, however, to have immunoglobulin A deposition in the mesangial area as well, supporting a diagnosis of immunoglobulin A nephropathy.

Pathologic descriptions of multiorgan involvement (i.e. muscle, nerve and spinal cord) CADASIL is rare. We document the CADASIL related arteriopathy in multiple visceral organs, including heart, lungs, spleen, testes, liver as well as paraspinal vessels, the significance of which is unclear. Despite diffuse arteriopathy in our patient, there was no evidence of organ infarction. The reason for this is unclear, though as outlined above the vascular involvement in the brain specifically affects cerebral autoregulation, causing leukoencephalopathy. As patients become more ill neurologically, renal or pulmonary disease might be attributed to declining health. However, involvement of other organs by CADASIL related arteriopathy must be considered.

\section{ACKNOWLEDGMENTS}

The authors thank Dr. Steven Chin for reviewing the autopsy pathology findings and Dr. David Pisani for help providing the MR images.

\section{Disclosures}

None by all authors.

\section{Statement of Authorship}

Dr. DeWitt: Contribution to writing the document, conceptualizing, and critical revision for intellectual content; Dr. Sangle: Contribution to writing and contribution to neuropathological review; Dr. Baringer: Critical revision of the manuscript for important intellectual content; Dr. Majersik: Critical review of genetic, laboratory, and family history.

\section{REFERENCES}

1. Tournier-Lasserve E, Iba-Zizen MT, Romero N, Bousser MG. Autosomal Dominant Syndrome with strokelike episodes and leukoencephalopathy. Stroke. 1991;22:1297-302.

2. Joutel A, Corpechot C, Ducros A, et al. Notch3 mutations in CADASIL, a hereditary adult-onset condition causing stroke and dementia. Nature. 1996;383:707-10.

3. Okeda R, Arima K, Kawai M. Arterial changes in cerebral autosomal dominant arteriopathy with subcortical infarcts and leukoencephalopathy (CADASIL) in relation to pathogenesis of diffuse myelin loss of cerebral white matter: examination of cerebral medullary arteries by reconstruction of serial sections of an autopsy case. Stroke. 2002;33:2565-9.

4. Tikka S, Mykkanen K, Ruchoux MM, et al. Congruence between NOTCH 3 mutations and GOM in 131 CADASIL patients. Brain. 2009;132:933-9.

5. Federico A, DiDonato I, Bianchi S, et al. Hereditary cerebral small vessel diseases: A review. J Neurol Sci. 2012;322:25-30. 